1 Three-dimensional morphological analysis of the dynamic digestive system in the green

2

3

4

$5 \quad{ }^{2}$ Research Institute for Electronic Science, Hokkaido University, Sapporo 0600812, Japan

6

7

8

9

10

\section{brittle star}

Daiki Wakita ${ }^{1}$, Keisuke Naniwa ${ }^{2}$, and Hitoshi Aonuma ${ }^{1,2 *}$

${ }^{1}$ Graduate School of Life Science, Hokkaido University, Sapporo 0600810, Japan

*Corresponding author: Hitoshi Aonuma, D.Sc.,

Research Institute for Electronic Science, Hokkaido University, Sapporo 0600812, Japan.

Tel/Fax: +81-117063832, Email: aon@es.hokudai.ac.jp 


\section{Abstract}

12 Brittle stars (Echinodermata: Ophiuroidea) digest a great diversity of food in their stomach,

13 which widely lies in the central disk. As for a possible digestive activity, the green brittle star

14 Ophiarachna incrassata is known to show a dynamic movement at the disk. This

15 phenomenon would deeply involve the morphological structure of the stomach. However,

16 past anatomical studies have shown the digestive system in two dimensions after wide

17 incision of the body wall anchoring the stomach. This methodology restrains us from

18 understanding how the stomach actually shapes inside a brittle star. We aim to visualize the

19 morphology of brittle stars' digestive system in a non-destructive and three-dimensional way,

20 with a comparison between a relaxed specimen and a specimen fixed at the very moment of

21 the disk's movement. Employing X-ray micro-computed tomography (micro-CT) and

22 introducing an instant freezing method with cryogenic ethanol, we found the stomach wholly

23 transformed during the movement. We here brought transparency to the in vivo position of gut

24 contents to hint the mechanism and digestive function of the movement. Our outcome

25 spotlights a dynamic digestive process in echinoderms and a widely applicable method for

26 probing into its relation with body structure.

27

\section{Keywords}

29 marine invertebrate, echinoderm, ophiuroid, stomach anatomy, feeding behavior, X-ray

30 micro-computed tomography, instant freezing 


\section{Introduction}

More than 2,300 species of brittle stars (Echinodermata: Ophiuroidea) are known worldwide and constitute the largest class among extant echinoderms (Stöhr et al. 2019). Diversity in feeding habits could be an explanatory factor for the current success of this group (Fontaine, 1965). Their food varies in sort and scale, from sediment or small organisms including diatoms, dinoflagellates, foraminifera, and copepods, to the whole or part of large organisms such as polychaetes, bivalves, crabs, fish, other echinoderms, and sessile algae

(Nagabhushanam \& Colman, 1959; Fontaine, 1965; Hendler \& Miller, 1984; Pearson \& Gage, 1984; Ambrose, 1993). Their radially extending arms play a role in capturing food (Fontaine, 1965; Warner, 1971; Reimer \& Reimer, 1975; Hendler \& Miller, 1984), and then internal digestive organs take center stage. Contrary to the various targets in feeding, brittle stars share the general structure of the digestive system. Its mouth is followed in order by buccal cavity, pharynx, esophagus, and stomach (Schechter \& Lucero, 1968). Lacking intestine and anus, it terminates with the stomach, which occupies a large space inside the disk — central part of the body (Smith, 1940; Schechter \& Lucero, 1968; Pentreath, 1971; Uchida \& Irimura, 1974). The stomach consists of a single sac radially dividing into 10 pouches: five radial (ambulacral) pouches and five interradial (interambulacral) pouches (Pentreath, 1969). We also read a contradicting statement that brittle stars' stomach totally has 15 swellings in a common context (Uchida \& Irimura, 1974). Interradial pouches are well-folded structure deeply lying between the bases of arms, whereas radial pouches are limited in narrow spaces over arms (Pentreath, 1969, 1971; Uchida \& Irimura, 1974). These pouches never extend into arms except the species Ophiocanops fugiens Koehler, 1922 (Fell, 1963). 

expansion and shrinkage can be recognized as a sort of peristaltic movement of the stomach.

Its unique coordinated patterns are explainable by assuming internal fluid flows, the way of which depends on the morphology of the disk. In particular, five-armed individuals make unsynchronized movements between five body parts, whereas those are well synchronized in a six-armed case-peculiar individual difference in brittle stars. Thus, in this phenomenon, the morphological structure of the stomach may be of great importance, although it is unclear how pumping transforms it. stars has been visualized only by two-dimensional sketches or tissue sections (Smith, 1940; body wall, which considerably distorts the morphology in focus. This issue arises from the

71 fragileness of digestive organs as well as its deep attachment to the body wall by collagen strands (Schechter \& Lucero, 1968; Uchida \& Irimura, 1974). The aim of our study is to 
78 six-armed specimen studied by Wakita et al. (2018), so as to internally validate the prior

79 assumption that this specific individual has six symmetrical units-previously it was made up

80 merely in external terms. The primary conclusion in our study is that pumping could

81 transform the entire stomach to help digestion in large brittle stars, with its coordinated

82 patterns apparently reflecting five- or six-fold symmetrical arrangement in internal

83 morphology.

84

85

86

87

88

89

90

91

92

93

94

95

96

97

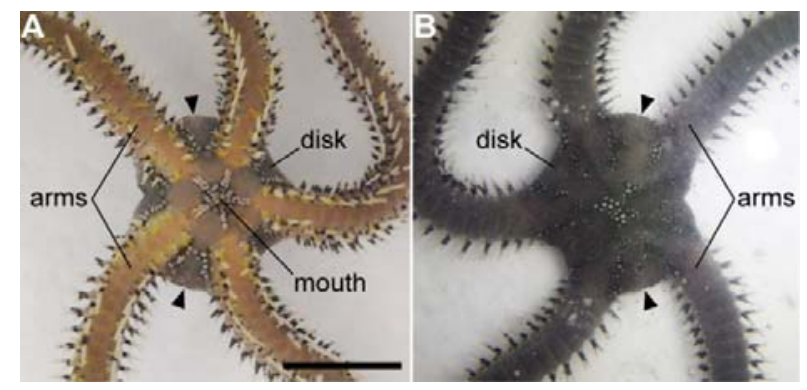

Fig. 1 Frozen individual at the moment of the dynamic movement "pumping" in the green brittle star Ophiarachna incrassata. (A) Oral view. (B) Aboral view. Photos were taken just after pouring $-80^{\circ} \mathrm{C}$ ethanol while we observed pumping at the disk. Arrowheads denote well-expanding portions. Scale bars represent $10 \mathrm{~mm}$. Scanned and segmented images of this specimen are shown in Fig. 3. Video of another individual's pumping is shown in Video S1.

Methods

\section{Animals}

Individuals of the green brittle star Ophiarachna incrassata were obtained commercially

(Aqua Shop Saien, Sapporo, Japan) and reared in aquariums $(600 \times 600 \times 600 \mathrm{~mm})$ filled with artificial seawater at $25-28^{\circ} \mathrm{C}$ with the salinity of $32-35 \%$ (TetraMarin Salt Pro, Tetra Japan Co, Tokyo, Japan). They were fed with dried krill (Tetra Krill-E, Tetra Japan Co, Tokyo, Japan). 


\section{$98 \quad$ X-ray micro-computed tomography}

99 With the aim to investigate relaxed and pumping bodies as well as five- and six-armed bodies,

100 we chose three individuals: (1) a five-armed individual a day after feeding, with the disk

101 diameter of $15 \mathrm{~mm}$ in a living anesthetized condition; (2) a five-armed individual a week after

102 feeding, with the disk diameter of $18 \mathrm{~mm}$; (3) a six-armed individual with the diameter of 25

$103 \mathrm{~mm}$, which had hardly shown feeding behavior for a few months. The six-armed one

104 corresponds to that studied by Wakita et al. (2018). For the relaxed case, the animals (1) and

105 (3) each were anaesthetized in $3 \% \mathrm{MgCl}_{2}$ solution for an hour at room temperature and then

106 fixed with Bouin solution-(1) for 10 days and (3) for five months - at $3^{\circ} \mathrm{C}$ with their arms

107 cut near the bases. For looking into the pumping body, the animal (2) was put in a styrofoam

108 box $(127 \times 157 \times 100 \mathrm{~mm})$ with $100-200 \mathrm{ml}$ of artificial seawater and then fed with a dried

109 krill. While we observed the rhythmic movement (pumping; c.f. Video S1), $-80^{\circ} \mathrm{C}$ ethanol

110 was poured onto the disk so that the animal (2) kept a momentary shape with expansion and

111 shrinkage (Fig. 1). After slowly shaking the box for $10 \mathrm{~min}$, the sample was put in Bouin

112 solution for a day at $3^{\circ} \mathrm{C}$ with their arms cut.

113 After fixation, all the samples (1)-(3) were dehydrated with ethanol series (70\%,

$11480 \%$, and $90 \%$ ) for two days each, and stained with $1 \%$ iodine diluted in ethanol for three

115 days at $3^{\circ} \mathrm{C}$ to enhance the contrast of tissues in later X-ray exposure (Metscher, 2009). They

116 were rinsed with $100 \%$ ethanol for a day at room temperature and then moved into $t$-butyl

117 alcohol liquidized with a water bath above $40^{\circ} \mathrm{C}$. After immersion in $t$-butyl alcohol for a day

118 twice at $26^{\circ} \mathrm{C}$, samples were superficially dried on tissues for several seconds and then put at

$119-20^{\circ} \mathrm{C}$ for $10 \mathrm{~min}$ so that instantly frozen $t$-butyl alcohol would keep the original morphology

120 as possible. They were freeze-dried by using a vacuum evaporator (PX-52, Yamato Ltd., 
121 Japan) with a cold alcohol trap (H2SO5, AS ONE, Japan). All chemicals were purchased from

122 Kanto Chemical Co. (Tokyo, Japan).

123 Samples were scanned on an X-ray micro-CT system (inspeXio, SMX-100CT,

124 Shimadzu Corporation, Kyoto, Japan), where X-ray source was operated at $75 \mathrm{kV}$ and $40 \mu \mathrm{A}$.

125 Scanned images were reconstructed and rendered by using VGStudio MAX ver. 2.2.6

126 (Volume Graphics, Heidelberg, Germany) with the voxel size of 10-50 $\mu \mathrm{m}$. For segmentation

127 of each sample using Amira ver. 2019.1 (Thermo Scientific, Waltham, USA), we traced the

128 inner surface — boundary with contrasting X-ray absorptivity—of the digestive cavity

129 beginning from the mouth. Note that segmentation was not available in regions where the

130 inner wall stuck to each other so that the cavity was too flat to be identified. We also

131 segmented gut contents, which were recognizable as highly absorptive areas inside the cavity.

132 3D animations were created with VGStudio MAX for slice images and Amira for segmented 133 images, which are given in Videos S2-4.

\section{Results}

136 The morphology of the digestive system, particularly the stomach, was well visualized by

137 segmenting the inner wall of the digestive cavity (Figs 2-4). In the five-armed specimen fixed

138 after anesthesia (Video S2), skeletal structure comprised five symmetrical sectors in

139 appearance (Fig. 2A). The cavity's surface was smoothly defined from the mouth to the

140 stomach (Fig. 2B), so we required less subjectivity in the segmentation (Fig. 2C-E). The

141 stomach comprised five larger interradial pouches and five smaller radial pouches (Fig. 2C).

142 As noted in Methods, no region in a totally flat cavity was segmented, hence the gaps forming

143 a cobweb-like structure could be interpreted as the missing flattened parts of the stomach, not 
144 representing there were many holes in morphology (Fig. 2C). The stomach was plain in the

145 aboral side (Fig. 2C,D) but well wrinkled in the oral (Fig. 2E). Viewed orally, a ridge could be

146 traced along each midline of two sorts of pouches, which descended into several branches

147 (Fig. 2E). Interradial pouches narrowed at the bases with their breadth increasing distally,

148 with the end being round so that we could trace smoothly between the oral and aboral surfaces

149 (Fig. 2C-E). Radial pouches were more flat, shaped along arm skeletal plates, and made distal

150 ends with rough and sharp edges (Fig. 2D,E). The distal parts of interradial pouches extended

151 until near the oral wall, whereas their bases and radial pouches were restricted aborally (Fig.

152 2D). The oral room at the center contained a jaw apparatus and its peripheral organs, which

153 would include the circumoral nerve ring and the water vascular system (Fig. 2A,B). In this

154 specimen, two large pieces of food were respectively observed at the aboral base of an

155 interradial pouch—might be partially shared by one adjacent radial pouch — and the oral end

156 of another interradial (Fig. 2C-E).
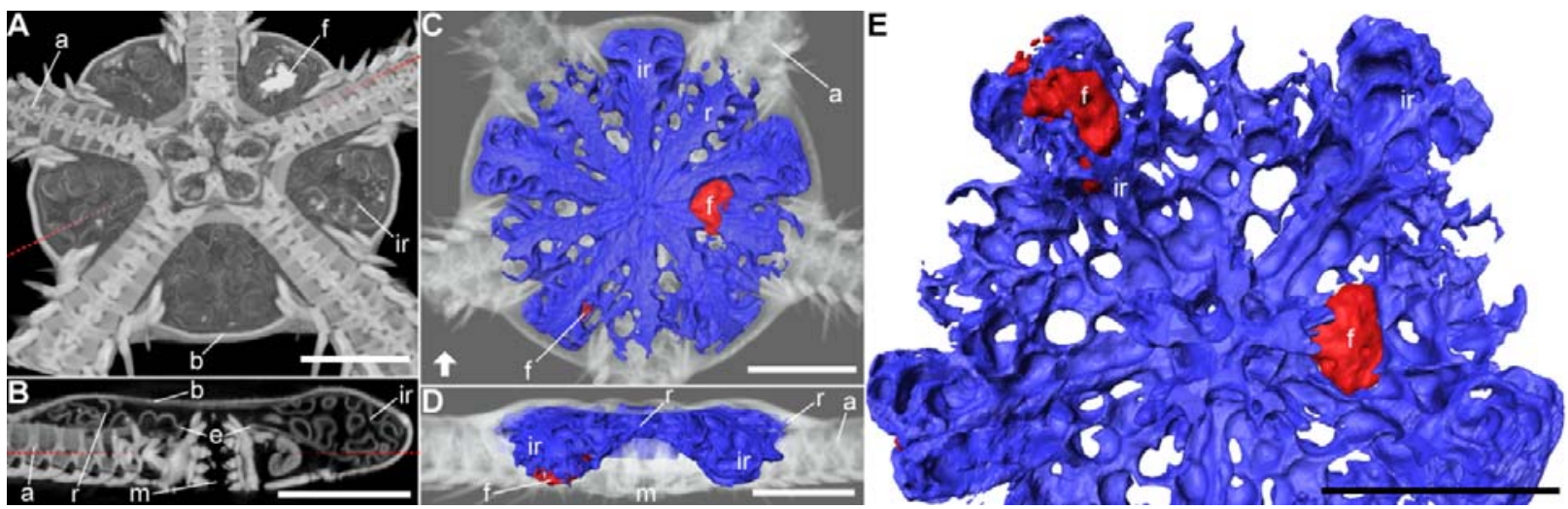

Fig. 2 Three-dimensional visualization of the digestive system in a five-armed relaxed

individual of the green brittle star Ophiarachna incrassata. Body structure was scanned with

$\mathrm{X}$-ray micro-computed tomography (micro-CT) and reconstructed in three dimensions, which

is displayed in grayscale. The inner surface of the digestive cavity beginning from the mouth 

from the observation that we saw no network-like segmentation denoting flattened patches

184 (Fig. 3C), contrasting to the relaxed one (Fig. 2C). Expansions were remarkable in interradial

is colored blue. Contents in the stomach are colored red. Regions where the inward cavity was totally flat were not segmented (not colored blue) with a technical limitation, which reflects the apparent holes of the stomach and the apparent exposure of gut contents. (A) Reconstructed images viewed from the oral side, sectioned at a plane shown in (B) by the dotted line. (B) Oral-aboral section on a plane indicated in (A) by the dotted line; the bottom is oral; slab thickness is $0.38 \mu \mathrm{m}$. (C) Aboral view of the segmented model. (D) Lateral view of the segmented model from the side indicated in (C) by the arrow; the bottom is oral; the grayscale images are truncated in the front for clarity. (E) Enlarged oral view of the segmented model. Abbreviations: a, arm skeleton; b, body wall; e, esophagus; f, food (gut content); m, mouth; ir, interradial pouch; r, radial pouch. Scale bars represent $5 \mathrm{~mm}$. Three-dimensional animation is shown in Video $\mathrm{S} 2$.

In the specimen frozen during pumping (Video S3), we found no noticeable damage due to the instant freezing method in external and internal morphology (Figs 1 and 3A). Besides, there seemed to be no large difference in the texture of the digestive wall in scanned images, compared to those observed in the relaxed one. We recognized a well-defined separation between the mouth and the stomach and a tight closure of the mouth (Fig. 3B; see the esophagus "e" and the mouth "m"), so we did not clearly identify the continuous space from the mouth opening. However, we had no ambiguity in segmenting the internal surface which was recognizable as the stomach's one (Fig. 3C-E) when comparing to the relaxed case (Fig. 2C-E). The stomach wall during pumping was smoothly fitted to the distorted body wall with less obvious folding (Fig. 3C). The globular shaping of the stomach was also upheld 
pouches, which directed toward the aboral and lateral sides (Fig. 3D). Meanwhile, radial

food lied almost at the center of the stomach in this specimen (Fig. 3C,E).
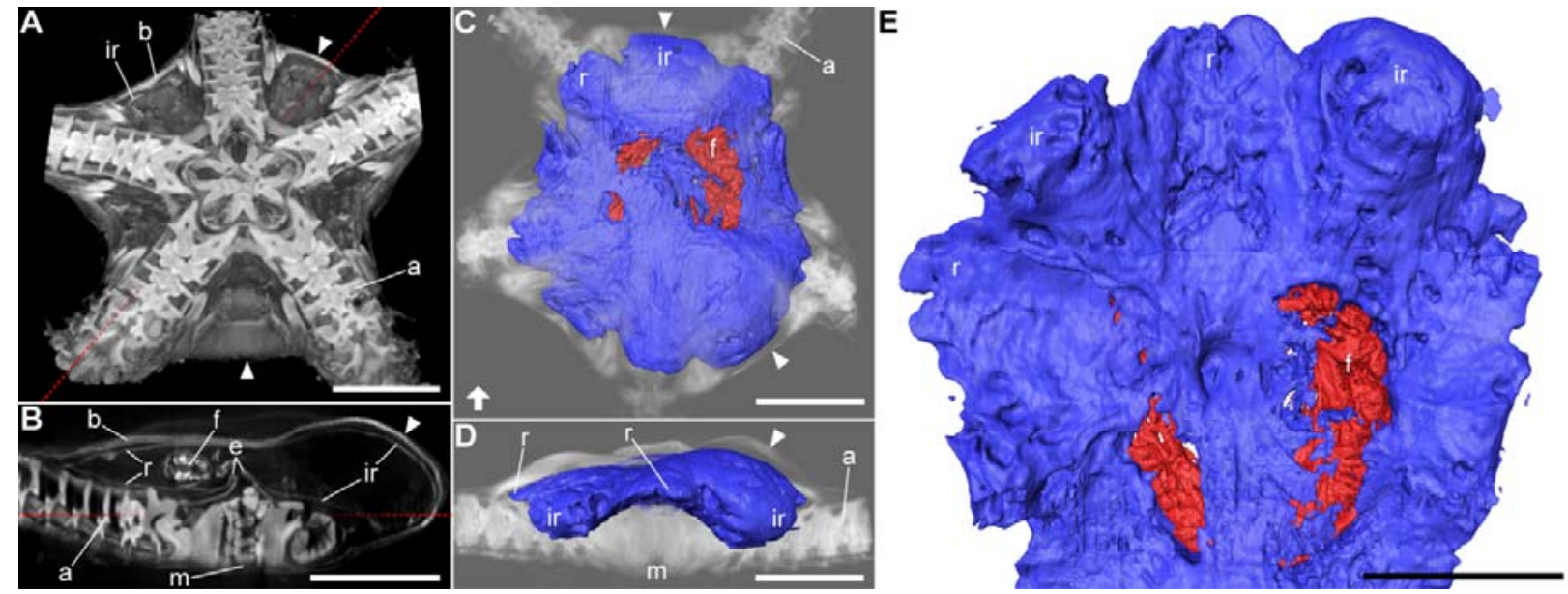

Fig. 3 Three-dimensional visualization of the digestive system in a five-armed individual

during the dynamic movement "pumping" in the green brittle star Ophiarachna incrassata.

Body structure of an instantly frozen individual shown in Fig. 1 was scanned with X-ray

micro-computed tomography (micro-CT) and reconstructed in three dimensions, which is

displayed in grayscale. The inner wall of the digestive cavity is colored blue, which was

identified in comparison with the relaxed specimen (Fig. 2). Contents in the stomach are

colored red. Regions where the inward cavity was totally flat were not segmented (not colored

blue) with a technical limitation, which reflects the apparent exposure of gut contents.

Arrowheads denote well-expanding portions. (A) Reconstructed images viewed from the oral side, sectioned at a plane shown in (B) by the dotted line. (B) Oral-aboral section on a plane 
indicated in (A) by the dotted line; the bottom is oral; slab thickness is $0.36 \mu \mathrm{m}$. (C) Aboral

view of the segmented model. (D) Lateral view of the segmented model from the side

indicated in $(\mathrm{C})$ by the arrow; the bottom is oral; the grayscale images are truncated in the

front for clarity. (E) Enlarged oral view of the segmented model. Abbreviations: a, arm

skeleton; b, body wall; e, esophagus; f, food (gut content); m, mouth; ir, interradial pouch; r, radial pouch. Scale bars represent $5 \mathrm{~mm}$. Three-dimensional animation is shown in Video S3.

apparently arranged in six-fold radial symmetry (Fig. 4A). As in the five-armed relaxed case,

212 flat as those of radial ones (Fig. 4B). This reduction was probably because this individual had

214 interradial pouches were less conspicuous (Fig. 4C) than the five-armed ones (Fig. 2C), the

215 two types could be distinguished when we saw the model from several angles (Fig. 4B,C). In

216 particular, interradial pouches hung down more orally (Fig. 4B) and showed ridged midlines

217 on the oral surface (Fig. 4C). Here we could count 12 pouches-six interradial and six radial

218 pouches (Fig. 4C).
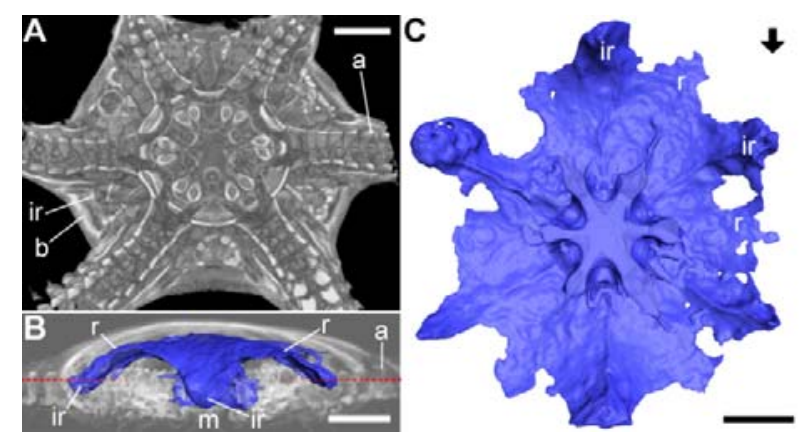

Fig. 4 Three-dimensional visualization of the digestive system in a six-armed relaxed 
221 individual of the green brittle star Ophiarachna incrassata. Body structure was scanned with X-ray micro-computed tomography (micro-CT) and reconstructed in three dimensions, which is displayed in grayscale. The inner wall of the digestive cavity beginning from the mouth is colored blue. Regions where the inward cavity was totally flat were not segmented (not colored blue) with a technical limitation. (A) Reconstructed images viewed from the oral side, sectioned at a plane indicated in (B) by the dotted line. (B) Lateral view of the segmented model from the side indicated in $(\mathrm{C})$ by the arrow; the bottom is oral; the grayscale images are truncated in the front for clarity. (C) Oral view of the segmented model. Abbreviations: a, arm skeleton; b, body wall; ir, interradial pouch; r, radial pouch. Scale bars represent $5 \mathrm{~mm}$. Three-dimensional animation is shown in Video S4.

\section{3} in brittle stars (Fig. 2, Video S2). The schematics were directly reconstructed from micro-CT scanned images, including less imagination than previous sketches. The second is introduction of a fresh methodology in micro-CT scanning, where we investigated a moving body's momentary shape made by instant freezing with cryogenic ethanol (Figs 1 and 3,

Video S3). The resultant snapshot provides a further understanding of the previously reported phenomenon, pumping (Wakita et al. 2018), as a possible digestive activity. The last is

240 internal inspection of the specific six-armed individual studied by Wakita et al. (2018) (Fig. 4,

241 Video S4). A supernumerary seemed to be simply a member of six equivalents, which helps a prebuilt assumption on pumping coordination. 
244 2C) as Pentreath (1969) reported, not supporting another literature writing the total number 15

245 (Uchida \& Irimura, 1974). Since the latter also mentions radial and interradial pouches, it

246 might be miswriting or another interpretation given the complex folding. The wrinkles

247 running on the oral surface would be a reflection in part of skeletal morphology and other

248 organs' anchoring, while probably being capable of a large amount of food by its extension. In

249 fact, the specimen shown in Fig. 3 accommodates a relatively large food ranging across

250 several pouches. Although the segmented stomach structure in Fig. 2 looks like a web

251 network, we can interpret that these holes as untraceable flat parts of the cavity so that

252 pouches connect with each other throughout the central space.

and 3) indicates that pumping dynamically transforms the stomach, where it is natural to

257 even in the pumping body (Fig. 3B,D). The mouth-stomach separation emerging in the

258 pumping specimen (Fig. 3B) can be defined as the constriction of esophagus, referring to

259 Schechter \& Lucero (1968). This observation and the tight closure of the jaw

260 apparatus—surrounding buccal cavity_(Fig. 3B) both would reinforce Wakita et al.'s (2018)

261 assumption that the total fluid volume is constant during pumping; there is no outward

262 leakage. Although their study built a water-connecting network with five nodes for the

263 five-armed case, there could be 10 rooms given the number of pouches (Fig. 2C). However,

264 the slits between the pouches were actually not deep as depicted in the pumping specimen

265 (Fig. 3C). With this texture, the five-fold arm skeleton would rather work as partitions (Fig.

266 3A,B), making it reasonable to represent five rooms influencing the major behavior of 
267 internal flows. This explanation could also apply to the six-armed case. The rigid skeletal

268 partitions symmetrically made by six arms (Fig. 4A) would be more dominant in fluid flow,

269 compared to the flexibly transformable stomach with 12 pouches (Fig. 4C). Our scan also

270 supports Wakita et al.'s (2018) explanation with six symmetrical nodes for this six-armed

271 specimen. We thus retain the explanatory power of the pumping network with five or six

272 symmetrical nodes-not 10 or 12 nodes in two different sizes.

Visibility in the original position of gut contents gives two suggestions for the

274 phenomenon pumping. The first is about its initiation. After a brittle star eats something, food

275 fragments would be seated at some pouches (Fig. 2C-E); even if a prey stays at the center, its

276 body shape would never weight equally among all the pouches (Fig. 3C). The contents thus

277 make the stomach morphology more asymmetric, which might trigger the initiation of a

278 pumping series as Wakita et al. (2018) let one interradial volume unequal at the beginning of

279 simulation. The second involves the purpose of pumping. In many animals including humans,

280 food transfers from the mouth to the anus in one direction; the linear structure guarantees that

281 nutrients are absorbed point by point. On the other hand, the digestive cavity of brittle stars

282 has neither unidirectional tracts nor the anus, so a piece might easily stick to a dead end — just

283 as shown in Fig. 2. Transformation of the stomach by pumping would give more opportunities

284 to displace the piece with it spreading a nutritious flow. This strategy would be effective in

285 large-sized species, where gut contents travel longer distances piece by piece. Therefore,

286 although we used the single species Ophiarachna incrassata, other large brittle stars are

287 supposed to exhibit pumping in a similar manner.

288 CT scanning technique for 3D visualization has been employed by several studies

289 on brittle stars. Landschoff \& Griffiths (2015) revealed how a brooding brittle star 
290 accommodates several juveniles inside its body, comparing two species; another was similarly

291 examined later (MacKinnon et al. 2017). In a taxonomic context, Okanishi et al. (2017)

292 described skeletal structure of a euryalid brittle star without dissolving its thick skin. Clark et

293 al. (2018) paid attention to the joint connection of vertebrae to understand the mobility of

294 arms in two species. Our study would carry a novelty in (1) focusing on the digestive system,

295 (2) comparing two behavioral conditions (relaxed v.s. pumping), and (3) comparing two

296 morphologically different individuals within a species (five-armed v.s. six-armed) in CT

297 scanned brittle stars. Notably, the snapshotting method for (2), where cryogenic ethanol is

298 poured onto a living animal, is widely applicable for the scanning purpose of body structure

299 during dynamic movements in echinoderms. These approaches give prominence to a dynamic

300 digestive process and its relation with body structure, which would be a hot clue to ethology,

301 ecology, and evolution in echinoderms.

302

303

\section{Acknowledgments}

304 This work was partly supported by JSPS KAKENHI (Grant Number 16KT0099), JST CREST

305 (Grant Number JPMJCR14D5), and Hokkaido University Frontier Foundation (Nitobe School

306 Financial Assistance), Japan.

307

308

\section{Competing interests}

309 The authors declare no competing financial interests.

310

311

\section{Author contributions}

312 D.W. and H.A. designed the study, D.W. conducted experiments, H.A. performed 
313 micro-computed tomography, K.N. conducted segmentation, D.W. drafted the manuscript and

314 prepared figures and videos, K.N. and H.A. revised the manuscript, and all the authors

315 approved the article.

316

317

\section{References}

318 Ambrose WG Jr (1993) Effects of predation and disturbance by ophiuroids on soft-bottom community structure in Oslofjord: results of a mesocosm study. Mar Ecol Prog Ser 97, 225-236. https://doi.org/10.3354/meps097225.

Clark EG, Hutchinson JR, Darroch SA, Mongiardino Koch N, Brady TR, Smith SA, Briggs DE (2018) Integrating morphology and in vivo skeletal mobility with digital models to infer function in brittle star arms. J Anat 233, 696-714. https://doi.org/10.1111/joa.12887.

Fell HB (1963) The phylogeny of sea-stars. Philos T R Soc B 246, 381-435. https://doi.org/10.1098/rstb.1963.0010.

Fontaine AR (1965) The feeding mechanisms of the ophiuroid Ophiocomina nigra. J Mar Biol Assoc UK 45, 373-385. https://doi.org/10.1017/S0025315400054904.

Frolova LT, Dolmatov IY (2006) Regeneration of the epithelial lining of the stomach after autotomy of a disk in the brittle star Amphipholis kochii (Lütken) (Echinodermata: Ophiuroidea). Russ J Mar Biol 32, 68-70. https://doi.org/10.1134/S106307400601010X. regenerating specimens of the brittlestar Amphipholis kochii. Biol Bull 218, 303-316. https://doi.org/10.1086/BBLv218n3p303. 
336 Hendler G, Miller JE (1984) Feeding behavior of Asteroporpa annulata, a gorgonocephalid

337 brittlestar with unbranched arms. B Mar Sci 34, 449-460. https://doi.org/10088/8751.

338 Lamarck JD (1816) Ordre second: radiaires échinodermes. In: Histoire Naturelle des Animaux

339 sans Vertèbres 2 (ed. Lamarck JD), pp. 522-568. Paris: Verdière, Libraire, quai des

$340 \quad$ Augustins, No. 27. https://doi.org/10.5962/bhl.title.12712.

341 Landschoff J, Griffiths CL (2015) Three-dimensional visualisation of brooding behaviour in

342 two distantly related brittle stars from South African waters. Afr J Mar Sci 37, 533-541.

343 https://doi.org/10.2989/1814232X.2015.1095801.

344 MacKinnon RB, Landschoff J, Griffiths CL (2017) Seasonality and 3D-visualization of

345 brooding in the hermaphroditic ophiuroid Amphiura capensis. Invertebr Biol 136,

346 146-158. https://doi.org/10.1111/ivb.12164.

347 Metscher BD (2009) MicroCT for comparative morphology: simple staining methods allow

348 high-contrast 3D imaging of diverse non-mineralized animal tissues. BMC Physiol 9, 11.

349 https://doi.org/10.1186/1472-6793-9-11.

350 Nagabhushanam AK, Colman JS (1959) Carrion-eating by ophiuroids. Nature 184, 285.

$351 \quad$ https://doi.org/10.1038/184285a0.

352 Okanishi M, Fujita T, Maekawa Y, Sasaki T (2017) Non-destructive morphological

353 observations of the fleshy brittle star, Asteronyx loveni using micro-computed

354 tomography (Echinodermata, Ophiuroidea, Euryalida). ZooKeys 663, 1-19.

355 https://doi.org/10.3897/zookeys.663.11413.

356 Pearson M, Gage JD (1984) Diets of some deep-sea brittle stars in the Rockall Trough. Mar

357 Biol 82, 247-258. https://doi.org/10.1007/BF00392406.

358 Pentreath RJ (1969) The morphology of the gut and a qualitative review of digestive enzymes 
in some New Zealand ophiuroids. J Zool 159, 413-423.

https://doi.org/10.1111/j.1469-7998.1969.tb03898.x.

361 Pentreath RJ (1971) Respiratory surfaces and respiration in three New Zealand intertidal

ophiuroids. J Zool 163, 397-412. https://doi.org/10.1111/j.1469-7998.1971.tb04540.x.

363

364

365

366

367

368

369

370

371

372

373

374

375

376

377

378

379

380

381

Reimer RD, Reimer AA (1975) Chemical control of feeding in four species of tropical ophiuroids of the genus Ophioderma. Comp Biochem Phys A 51, 915-927. https://doi.org/10.1016/0300-9629(75)90075-4.

Schechter J, Lucero J (1968) A light and electron microscopic investigation of the digestive system of the ophiuroid Ophiuroiderma panamensis (brittle star). J Morphol 124, 451-481. https://doi.org/10.1002/jmor.1051240405.

Smith J (1940) The reproductive system and associated organs of the brittle-star Ophiothrix fragilis. J Cell Sci 2, 267-309.

Stöhr S, O’Hara T, Thuy B (2019) World Ophiuroidea Database. http://www.marinespecies.org/ophiuroidea. Accessed 29 April 2019. https://doi.org/10.14284/358.

Uchida T, Irimura S (1974) Ophiuroidea. In: The Systematic Zoology $8 B$ (ed. Uchida T), pp 142-207. Tokyo: Nakayama Book Company.

Wakita D, Hayase Y, Aonuma H (2018) Five breaks synchrony while six keeps synchrony: individual difference in the coordinated pattern of five- and six-armed brittle stars. BioRxiv, 340471. https://doi.org/10.1101/340471.

Warner GF (1971) On the ecology of a dense bed of the brittle-star Ophiothrix fragilis. J Mar Biol Assoc UK 51, 267-282. https://doi.org/10.1017/S0025315400031775. 


\section{Supporting Information}

384 Video S1. Rhythmic movement "pumping" in a five-armed individual of the green brittle star

385 Ophiarachna incrassata.

386 Video S2. 3D animation of the images shown in Fig. 2 (five-armed relaxed individual).

387 Video S3. 3D animation of the images shown in Fig. 3 (five-armed pumping individual).

388 Video S4. 3D animation of the images shown in Fig. 4 (six-armed relaxed individual). 